DE

M E D I C I N A

T R O P I C A L

$\mathrm{DE}$

S ÃO PAULO

JOURNAL OF THE SÃO PAULO INSTITUTE OF TROPICAL MEDICINE

${ }^{1}$ Complexo Hospitalar Padre Bento de Guarulhos, Departamento de Dermatologia, Guarulhos, São Paulo, Brazil

Correspondence to: Mariana de Freitas Valente

Complexo Hospitalar Padre Bento de

Guarulhos, Departamento de Dermatologia,

Av Emilio Ribas, 1819, CEP 07051-000,

Guarulhos, SP, Brazil

Tel: +55 11 2463-5690

E-mail: marianadfvalente@gmail.com, marivalente09@hotmail.com

Received: 22 April 2020

Accepted: 10 August 2020

\section{Disseminated cutaneous sporotrichosis: unusual presentation in an alcoholic patient}

\author{
Mariana de Freitas Valente ${ }^{(1)}$, Amanda Bertazzoli Diogo', Vitoria Flores \\ Culau Merlo', José Roberto Pereira Pegas ${ }^{1}$
}

\begin{abstract}
Sporotrichosis is an implantation mycosis highly prevalent in Brazil, associated with soil activities and contact with infected animals. It has numerous clinical manifestations and its disseminated cutaneous form is uncommon, found in approximately $4 \%$ of cases and usually related to immunosuppressive conditions. We report an alcoholic 56-year-old male patient with no other comorbidities, presenting with multiple cutaneous nodules and ulcers. The patient was diagnosed with disseminated cutaneous sporotrichosis based on the isolation and identification of Sporothrix spp. The patient was initially treated with amphotericin B with satisfactory results and then with itraconazole.
\end{abstract}

KEYWORDS: Sporotrichosis. Alcoholism. Immunosuppression. Disseminated cutaneous sporotrichosis. Sporothrix spp.

\section{INTRODUCTION}

Sporotrichosis is an implantation mycosis that affects the cutaneous and subcutaneous tissues and, eventually, mucous membranes and other organs, especially in immunocompromised patients. In the past, it was referred as a subcutaneous mycosis, but the term "subcutaneous" should be avoided because sporotrichosis, like micetoma, may involve several organs sites beyond the subcutaneous tissues. Gardening activities, soil handling and contact with infected animals are major risk factors for the disease's transmission. The most common clinical presentation is the cutaneous lesion with lymphatic involvement; the disseminated cutaneous form is uncommon and more associated with HIV infections. The diagnosis is confirmed by isolation and identification of the etiological agent through culture or histopathology, and the recommended treatment involve antifungals as itraconazole and amphotericin $\mathrm{B}^{1,2}$. Although an unusual presentation of sporotrichosis, reports of the disseminated cutaneous forms are necessary so that similar cases can be recognized and promptly treated, avoiding the involvement of other organs and unfavorable outcomes.

\section{CASE REPORT}

A 56-year-old male Brazilian patient from Itabuna, Bahia and resident of Guarulhos, Sao Paulo presented with an erythematous nodule on his left wrist that appeared two months before. The nodule ulcerated, and similar disseminated cutaneous lesions appeared after the first one, with progressive growth. The patient was unemployed and had a history of alcoholism but no other medical 
conditions; he denied contact with animals or plants. On physical examination, he had numerous erythematous and ulcerated nodules, ulcers with well-framed borders, purulent and crusted lesions, especially in the cephalic segment (Figure 1). He had a regular general condition, with no other abnormalities on physical examination.

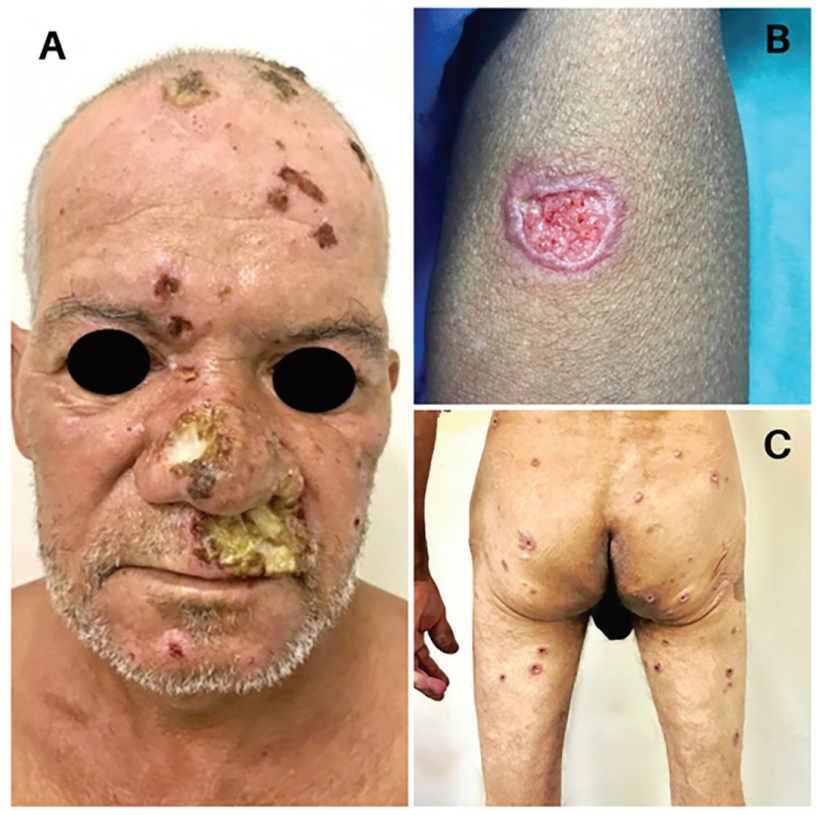

Figure 1 - A) erythematous and ulcerated nodules, ulcers with well-framed borders, purulent and crusted lesions on the face and the scalp; B) well limited ulcer with well-framed border on the left arm; C) erythematous and ulcerated nodules scattered on the skin.

Initially, the main hypotheses were cutaneous leishmaniasis, disseminated histoplasmosis, malignant syphilis and paracoccidioidomycosis. Laboratory tests were requested, including serology and skin biopsy. The patient was hospitalized and started antibiotic therapy with ciprofloxacin and clindamycin due to a secondary bacterial infection, while awaiting the test results. Serology for hepatitis, syphilis and HIV were negative. He had a mild anemia and a slight alteration of the liver enzymes, which suggested some degree of previous liver injury possibly due to his alcoholism. Involvement of other organs was investigated, but exams did not show any abnormalities. Skin biopsy and mycological examination showed a positive culture for Sporothrix spp (Figure 2A). Histopathology showed superficial and deep granulomatous dermatitis with suppuration, multinucleated giant cells (Figure 2C) and PAS staining showed the presence of ovoid and "cigar-shaped" structures (Figure 2B).

The patient was diagnosed with disseminated cutaneous sporotrichosis; treatment with amphotericin B deoxycholate $50 \mathrm{mg} /$ day $(1 \mathrm{mg} / \mathrm{kg} / \mathrm{day})$ was started and resulted in

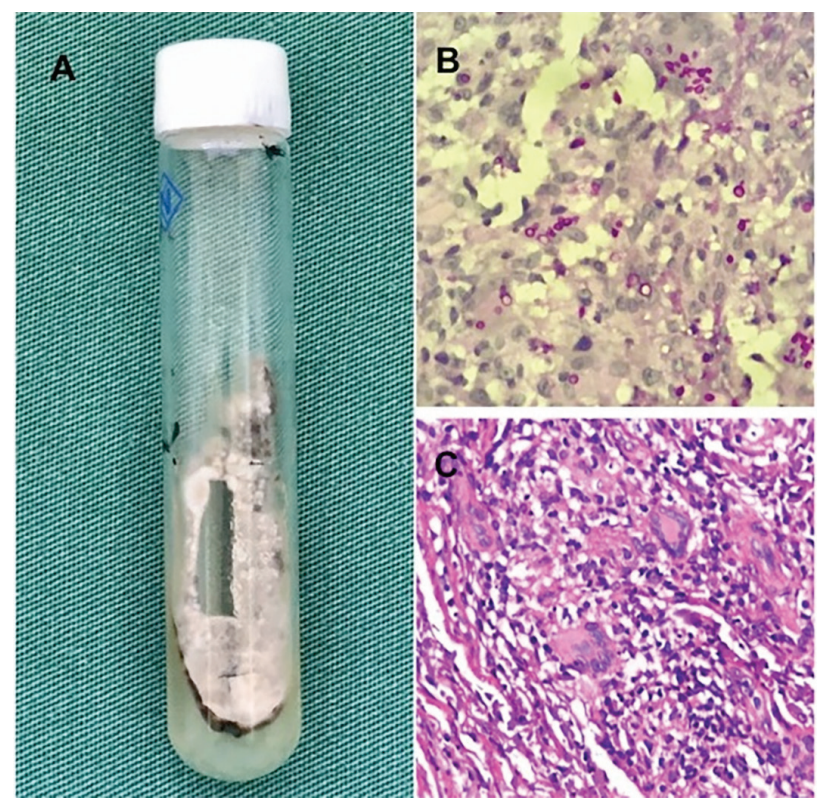

Figure 2 - A) obverse of culture - cream color with blackened halo; B) presence of ovoid, round and cigar-shaped structures (PAS $400 \mathrm{x}$ ); C) granulomatous dermatitis with multinucleated giant cells $(400 \mathrm{x})$

progressive improvement of the lesions. However, after 15 days, the patient presented acute renal injury requiring discontinuation of amphotericin B and institution of appropriated clinical measures. After recovery, itraconazole $200 \mathrm{mg} /$ day was introduced with careful liver monitoring and, until this case report was submitted, the patient had been using the medication for 12 weeks with excellent tolerance and response (Figure 3).

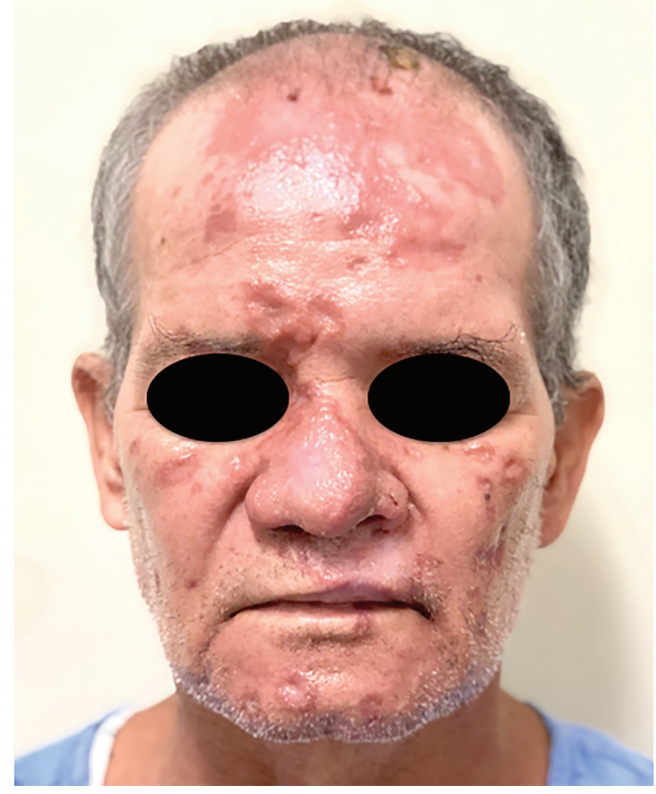

Figure 3 - Substantial improvement of lesions after treatment. 


\section{DISCUSSION}

Sporotrichosis is the most prevalent implantation mycosis, reaching epidemic levels in Brazil. Infection is attributed to the contact with infected animals and handling of contaminated soil, plants, or materials ${ }^{1}$. Two main routes are involved in its transmission, a sapronotic one that implies direct contact with the soil and decomposing organic matter; and the other, a zoonotic one with the participation of felines, especially cats in Brazil ${ }^{2}$.

The disease is caused by the dimorphic fungus Sporothrix spp. However, there is a difference in the incidence of etiological agents according to the geographic distribution of the infection. In North America and part of South America S. schenckii is the predominant species (89\%), while in the South and Southeast Brazilian regions, S. brasiliensis $(88 \%)$ is the main etiological agent of human and animal sporotrichosis ${ }^{3,4}$. There are genotyping methods to identify the pathogenic species in Sporothrix such as DNA sequencing - PCR (Polymerase Chain Reaction) technique ${ }^{2}$. The species identification should be always performed, but unfortunately, in our case the test was unavailable.

Sporotrichosis has been classified into four clinical forms: cutaneous-lymphatic, fixed, disseminated and extracutaneous. Disseminated cutaneous sporotrichosis is an uncommon type of presentation, found in approximately $4 \%$ of cases and usually related to immunosuppression conditions such as infections, diabetes, alcoholism and medications ${ }^{1}$. In alcoholism, ethanol inhibits the Kappa B factor activation and is associated with decreased TNF (tumor necrosis factor) and IL $\beta$ (interleukin beta) causing immune deterioration related to cellular immunity and macrophage dysfunction. However, the exact physiopathology through which ethanol exerts its immunosuppressive effects are still unclear ${ }^{5}$. Most cases of the disseminated form are described in HIV-positive patients ${ }^{6}$. The patient of this case report was tested for HIV with a negative result, so alcoholism was considered his major risk factor for the development of the disseminated form.

Disseminated cutaneous sporotrichosis is characterized by multiple and diffuse lesions on the tegument, such as plaques, necrotic and warty lesions located in different body segments ${ }^{7}$. Mucosal involvement is rare ${ }^{2}$ and the most commonly involved mucosa is the ocular one which was not affected in this reported case.

The gold standard for sporotrichosis diagnosis is culture and the clinical specimen may be obtained by biopsies, aspirates, swabs etc. Culture has a white or cream color with a blackened halo, such as the one observed in this case. Histopathology shows diffuse chronic granulomatous dermatitis, often with a central abscess and multinucleated giant cells. The presence of asteroid bodies (i.e. the Splendore-Hoeppli phenomenon) may point to the diagnosis, although it can be found in other diseases, as well. This finding, however, was not observed in this case. Direct microscopic examination with potassium hydroxide may show rounded "cigar-shaped" oval structures, which are more commonly seen in immunocompromised patients ${ }^{1}$, as in the reported case.

Treatment involve antifungal drugs as itraconazole, potassium iodide, terbinafine, and amphotericin B. Itraconazole, which is a fungistatic drug, is considered the first option of treatment due to its effectiveness, safety, and dosage convenience. Disseminated forms affecting deep organs may require a more aggressive amphotericin B treatment. The recommended dose is 0.5 to $1 \mathrm{mg} / \mathrm{kg} / \mathrm{day}$ (total dose 1-2 grams), followed by itraconazole as a maintenance schedule until clinical remission ${ }^{2}$; immunocompromised patients may need more prolonged treatment ${ }^{6}$. Our patient used this scheme with an excellent result; we started treatment with amphotericin $\mathrm{B}$, in this case due to the high number of lesions, and as a strategy to get a fastest improvement as the patient was very afflicted especially in relation to the lesions on his face. However, the disseminated cutaneous form is not a formal indication for the use of amphotericin $\mathrm{B}^{2}$.

The increased incidence of sporotrichosis in Brazil has led to an increase in the number of atypical and severe cases with lesions morphologically different from the usual ones, with both, generalization of lesions and extracutaneous involvement ${ }^{2}$. In the reported case, we believe that alcoholism and the delayed diagnosis and treatment resulted in the rapid and unusual dissemination of the lesions.

\section{ACKNOWLEDGMENTS}

We thank Dra Ana Maria Yoshino Bonifaci for helping with the histopathological exams to diagnose the case.

\section{AUTHORS' CONTRIBUTIONS}

Mariana de Freitas Valente: investigation, methodology, validation, visualization, writing; Amanda Bertazzoli Diogo: data curation, validation, visualization, writing; Vitoria Flores Culau Merlo: investigation, validation, visualization, writing; José Roberto Pereira Pegas: investigation, project administration, supervisión, validation, visualization. All the authors read and approved the final version of the manuscript.

\section{CONFLICT OF INTERESTS}

The authors declare no conflict of interests 


\section{REFERENCES}

1. Fava SC, Costa MA. Esporotricose. In: Belda Junior W, Di Chiacchio N, Criado PR, editores. Tratado de dermatologia. $3^{a}$ ed. São Paulo: Atheneu; 2018. p.1643-6.

2. Orofino-Costa R, Macedo PM, Rodrigues AM, BernardesEngemann AR. Sporotrichosis: an update on epidemiology, etiopathogenesis, laboratory and clinical therapeutics. An Bras Dermatol. 2017;92:606-20.

3. Rodrigues AM, Teixeira MM, de Hoog GS, Schubach TM, Pereira SA, Fernandes GF, et al. Phylogenetic analysis reveals a high prevalence of Sporothrix brasiliensis in feline sporotrichosis outbreaks. PLoS Negl Trop Dis. 2013;7:e2281.
4. Rodrigues AM, de Hoog G, Zhang Y, Camargo ZP. Emerging sporotrichosis is driven by clonal and recombinant Sporothrix species. Emerg Microbes Infect. 2014;3:e32.

5. Benvegnú AM, Stramari J, Dallazem LN, Chemello RM, Beber AA. Disseminated cutaneous sporotrichosis in patient with alcoholism. Rev Soc Bras Med Trop. 2017;50: 871-3.

6. Wong SM, Tang JJ. Disseminated sporotrichosis in a patient with a past history of lepromatous leprosy: a case report. Med Mycol. 2012;50:404-6.

7. Bonifaz A, Tirado-Sánchez A, Paredes-Solís V, Cepeda-Valdés R, González GM, Treviño-Rangel RJ, et al. Cutaneous disseminated sporotrichosis: clinical experience of 24 cases. J Eur Acad Dermatol Venereol. 2018;32:e77-9. 